Pensamiento Crítico $N^{\circ} 16$, pp. 69-77

\title{
Crisis financiera internacional: enfoque en instrumentos y riesgos
}

\author{
Carlos Palomino Selem
}

\section{RESUMEN}

La crisis financiera 2008 fue muy diferente respecto a otras crisis financieras porque en esta el mercado de capitales tenía instrumentos financieros muy sofisticados que tuvieron dos características: por un lado, permitieron estructurar deuda a través de la cual los instrumentos de deuda fueron segmentados en capital e intereses que permitieron diversificar riesgo y bajarlo. Por otro lado, los acuerdos incluyeron seguros conocidos como CDS (credit default swap) los cuales intercambian anualidades que apoyan en caso la deuda caiga en insolvencia. Este esquema dentro de un contexto de globalización potenció y profundizó la crisis.

Palabras Claves: Riesgo de no pago, títulos respaldados con activos, títulos respaldados con hipotecas, obligaciones de deuda colateralizada, vehículo financiero, seguro de crédito contra riesgo de no pago, flexibilización cuantitativa. 


\title{
Pensamiento Crítico $\mathrm{N}^{\circ} 16$
}

\begin{abstract}
The financial crises 2008 was very different respect to others financial crises because in this the market capitals had financial instruments very sophisticated that had two characteristic: one side, permitted debt structuring through which the debt instruments was segmented in capital and interest, that allowed risk diversification and low it. Other side, the agreements included embedded "insurances" known like CDS (credit default swap) which are exchange annuity payments that supported in case the debt fall in default. This scheme into of a context of globalization potentiated and deepened the crises.
\end{abstract}

Keywords: Default risk, asset backed securities, mortage backed security, collateralized debt obligation, financial vehicle, creddit default swap, quantitative easing.

\section{Introducción}

En el presente documento presentamos un elemento importante que distinguir de la crisis financiera del 2008 respecto a otras crisis financieras como la del lunes negro de 1987, la burbuja de Japón el año 1989, o la de Long Term Capital Management (LTCM), un fondo de cobertura (fondo hedge) donde participaron como figuras relevantes dos Premios Nobel de Economía. La diferenncia se encuentra en el empleo de sofisticados instrumentos financieros que subyacieron en ella y profundizaron su avance y multiplicaron su expansión.

En primer término, presentaremos las hipótesis que planteamos respecto al rol de los instrumentos financieros en la crisis del 2008. En segundo lugar, presentaremos el marco teórico que fundamenta el impacto de los instrumentos financiero en la crisis. Finalmente, en la tercera parte de este documento, mostraremos los resultados vistos desde las perspectivas del marco teórico.

Queremo por último señalar, que el presente trabajo, es un planteamiento inicial, resultado del proyecto de investigación llevado acabo el año 2011 para el Instituto de Investigación Económica. 


\section{Carlos Palomino Selem}

\section{Las hipótesis}

La crisis financiera en USA, sigue el perfil de importantes crisis financieras recientes como las de algunos países del Asia (Parodi 2001) donde se produce un desbalance importante de deuda realizada, pero insolvente de ser cancelada, lo cual trajo consigo un alto riesgo de default.

A diferencia de las crisis anteriores, los instrumentos financieros empleados no se basaron en instrumentos básicos como acciones y/o bonos; sino, en instrumentos más sofisticados como las titulizaciones o denominas en ingles como asset backed securities (ABS), en particular los instrumentos tutilizados provenientes de deuda hipotécaria (mortgage backed security-MBS-), y otros más complejos como los Collateralized Debt Obligation (CDO) que podían incluir deuda hipotecaria como otro tipo de deuda, incluyendo lo que se conoce como segmentación de instrumentos (strip) que permiten fraccionar la deuda en dos partes; una es el principal (capital), la misma que puede negociarse en un vehículo financiero, independiente de los intereses que trae consigo; los cuales, se pueden por tanto separar de la deuda original, y negociar por separado en vehículos financieros como los $\mathrm{CDO}$.

Un aspecto importante que resaltar es que estos MBS o CDO permitían "empaquetar" deuda hipotecaria de muy baja calidad crediticia por su alto riesgo de insolvencia (deafualt) conocida como Subprime. Por consiguiente, quienes la compraban un CDO podían desconocer la composición de estos, o en su defecto, considerar que el "paquete" por su composición podía compensar pérdidas con ganancias y resultar con retornos netos favorables.

La titulización de instrumentos consiguió que el capital prestado por los bancos se realizará de forma inmediata por estos, y se convirtier en liquidez para estas instituciones, de manera que la maduración o plazo de la deuda se redujera de forma acelerada, sin emplear protecciones del tipo Callable

Acompañó a este esquema un modelo de "seguros" financieros novedosos, conocidos con el nombre de Credit Default Swap (CDS), la cuales son unas permutas financieras que intercambiaban deuda proveniente de los tenedores de CDO hacia las compañías aseguradoras, las mismas que en caso de default tendrían que afrontar 


\section{Pensamiento Crítico $\mathrm{N}^{\circ} 16$}

la deuda impaga, a cambio de una serie de pagos periódicos denominados (spread) realizados por los tenedores de deuda hacia los aseguradores, de manera que si la deuda cayese en riesgo de default los aseguradores se comprometían a pagar dichas deudas. Lo novedoso del CDS en que para su emisión no era necesario que existiese una deuda de por medio, a este tipo de CDS, se le denominaba CDS descubierto o Naked.

Este modelo de seguros incentivó la negociación de $\mathrm{CDO}$, los cuales fueron motivo de la caída de Lehman Brothers, principal agente tomador de CDO, y el rescate de la importante aseguradora AIG, quien cubría los CDO con CDS. Este fue el esquema detonador de la crisis financiera del 2008.

\section{El marco teórico}

Consideramos que en el plano estratégico la realización de la rentabilidad de la banca se encuentra en la reducción del plazo de maduración de un activo provenientes de los; dado que las cuotas de los préstamos se convierten en el cash flow de los mismos. Esto permite mejorar la liquidez de la firma bancaria mejorando el nivel de apalancamiento bancario como lo exige los acuerdos de Basilea.

En efecto, si se define el ratio de apalancamiento bancario ( $\mathrm{r}$ ) como:

$$
\mathrm{r}=\mathrm{L} / \mathrm{Pat} \quad---(1)
$$

donde $\mathrm{L}=$ préstamos y Pat $=$ patrimonio bancario. Un objetivo estratégico para los bancos es:

Optimizar [ L/ Pat ] --- (2)

Para ello el total de activos (A) de los bancos se puede modelar como:

$$
\mathrm{A}=\mathrm{Li}+\mathrm{R}+\mathrm{L}---
$$

siendo $\mathrm{Li}=$ caja y/o liquidez, y $\mathrm{R}=$ encaje en el banco central. Donde $-\partial \mathrm{L} / \partial \mathrm{t}=$ $\partial \mathrm{Li} / \partial \mathrm{t}$, ( $\mathrm{t}$ es el tiempo). Los bancos buscan reducir el nivel de $\mathrm{L}$ que se transforma en 


\section{Carlos Palomino Selem}

liquidez de manera que $\mathrm{L}$ alcance el nivel de préstamos óptimo $\mathrm{L}=\mathrm{L}^{*}$ en el portafolio de la ecuación (3).

Instrumentos titulizados como los MBS tuvieron como objetivo alcanzar dicho nivel; optimizando el portafolio para L:

Optimizar [ $\mathrm{Li}(\mathrm{Ke})+\mathrm{R}+\mathrm{L}(\mathrm{i})]$--- (4)

sujeto a:

$A=A^{*}($ constante)

R constante e

$$
\mathrm{i}=\mathrm{iR}+\operatorname{spread}(\mathrm{s})
$$

$\mathrm{s}$ spread de riesgo, i tasa de interés de los bancos, iR tasa de referencia del banco central.

El resultado sería $\partial \mathrm{Li} / \mathrm{K} e=\partial \mathrm{L} / \mathrm{i}, \mathrm{Ke}$ rentabilidad para los accionistas del banco.

El desequilibrio se presenta cuando $\partial \mathrm{Li} / \mathrm{Ke}>\partial \mathrm{L} / \mathrm{i}$ y el rendimiento de los préstamos es menor al rendimiento de la liquidez lo cual obliga a promover vehículos financieros como MBS o CDO.

La suma de $\mathrm{L}$ transformados a MBS operacionalizada como $\triangle \mathrm{MBS}=\Delta \mathrm{L}$, se permite expresar la relación siguiente:

$$
\Delta \mathrm{MBS}=-\partial \mathrm{L} / \partial \mathrm{t}=\partial \mathrm{Li} / \partial \mathrm{t}
$$

donde el $\partial \mathrm{L} / \partial \mathrm{t}$ se transfiere como $\triangle \mathrm{MBS}$.

En el caso de los CDO la deuda proviene de los mercados de capitale $\left(\mathrm{M}_{4}\right)$ en caso de bonos o del mercado de dinero en el caso de instrumentos de corto plazo, pero 


\section{Pensamiento Crítico $\mathbf{N}^{\circ} 16$}

puede incluir deuda subprime que se empaqueta en dentro de un CDO.

$$
\begin{aligned}
& \partial \mathrm{CDO} / \partial \mathrm{t}=\partial \mathrm{M}_{4} / \partial \mathrm{t} \quad---(6 \mathrm{~A}) \\
& \text { y } \quad \partial \mathrm{M}_{4} / \partial \mathrm{t}>\partial \mathrm{M}_{1} / \partial \mathrm{t} \quad---(6 \mathrm{~B})
\end{aligned}
$$

M1 oferta monetaria, además:

$$
\partial 2 \mathrm{M}_{4} / \partial 2 \mathrm{t}>\partial 2 \mathrm{M}_{1} / \partial 2 \mathrm{t}
$$

La expresión (7) indica que el sistema se biene endeudando a un ritmo mayor que el crecimiento de la oferta monetaria en el tiempo y el riesgo de default incrementa; requiriendo una mayor monetización.

Cuando los MBS y CDO cayeron en default,estos finalmente se tuvieron que financiar con un incremento de la base monetaria (B) del Sistema de la Reserva Federal -Fed- (monetización) (ver gráfica N. 1). Lo cual, se observó al momento de enfrentar la crisis con las políticaas de flexibilización cuantitativa (Quantitative Easing) posteriormente distorsionó la curva de rendimiento de los instrumentos del tesoro USA, debido a que las tasas de interés del tramo corto de la curva de rendimiento de los treasuries cayeron, dado que la $\Delta \mathrm{B}$ se llevó a cabo con una activa participación del Fed, el cual motivó el incremento de los precio de dichos instrumento y su caida en la tasa de interés.

\section{Resultados}

Las estimaciones respecto a las pérdidas en la crisis financiera son dificiles de establecer, esto debido al hermetismo con que se ha tratado el tema. Auque se podría nombrar algunas cifras que darían una idea de las magnitudes que se alcanzarón las deudas y pérdidas.

\subsection{Resultados sobre la expansión de la crisis financiera}

La primera parte de la crisis financiera, es expresa en la expansión de ésta acompañada por los vehículos financieros (MBS y $\mathrm{CDO}$ ) que la permitireron; lo cual sobrepasó montos importantes. En efecto, de acuerdo a Tully (2008) las cuentas 


\section{Carlos Palomino Selem}

incobrables a marzo del 2008 de los bancos de inversión de USA superaban los 230 mil millones de USD en préstamos de hipotécas y otros bienes; y a nivel mundial, de acuerdo al Lotta (2008), el contagio alcanzó más de \$1,000,000,000,000.

En marzo del 2009, cuando el Índice Industrial Promedio Dow Jones (DJI) tocó su nivel más bajo de la primera década del siglo XXI, alrededor de los 6,626; El FMI reportó que los mercados financieros de Europa, USA y Japón registrarían pérdidas entre 2007 y 2010 , de $\$ 4,1$ billones, hasta ese instante el sector bancario había perdido \$1 billón (WSJ 2009).

De otra parte, los CDS que aseguraban los $\mathrm{CDO}$ y que tuvo su principal agente asegurador en la compañía de seguros AIG Group, la aseguradora de Lehman Brothers, el banco de inversión que representaba alrededor del $80 \%$ de los CDS del mercado, recibió dos programas de facilidad, uno por USD 85 mil millones y otro por USD 37.8 mil millones; en total, 122.8 mil millones (Navarro 2009).

\subsection{Resultados sobre los requerimiento de rescate de la crisis financiera}

En el mismo documento del FMI, con la finalidad de alcanzar los niveles de capitalización bancaria anteriores a la crisis financiera, los bancos necesitarían recaudar $\$ 875.000$ millones en 2009. Respecto a este tema, le correspondió a la Fed emplear dos instrumentos; uno de ellos, el crédito interno al sistema financiero; el segundo, comprar instrumentos del tesoro USA. Según Hilsenrath \& Blackstone (2009), para el 2009 la FED, anunció que compraría US\$300.000 millones en valores del Tesoro USA para el rescate, y que además desembolsaría US $\$ 1,25$ billone.

\section{Conclusiones}

En el proceso de inestabilidad financiera desde mediados del 2007 se observa que los instrumentos financieros amplificaron la profundidad que pudo tomar la crisis de emplear sólo los instrumentos financieros básicos como los bonos, sea cual fuere la modalidad de estos (ejemplo: callable). Los instrumentos permitían que los riesgos se transfieran a distintos instrumentos tanto mediante vehículos financieros como innovadoras "garantías" creadas como son los CDS. 


\section{Pensamiento Crítico $\mathrm{N}^{\circ}$ I6}

Otro rol importante, tocó a la globalización financiera, la misma que mediante el canal de distribución de los instrumentos (comercialización), permitía que estos se negociaran en plazas distintas a la del lugar de origen de donde provenían, esto mediante los mecanismos de negociación electrónica.

Por el lado, de los bancos centrales, particularmente de la Fed, su principal mecanismo de control quedó en manos de la administración de la tasa de interés de referencia, dado que la Fed, se desentendió de su papel como regulador del riesgo crediticio que una vez se le asignó. Esta administración de la tasa de interés promovió más que controlar el proceso financiero; pero poco se puede hacer cuando de préstamos bancarios se pasó al mercado de capitales.

Finalmente, las secuelas de la crisis financiera, por el sector real a través del empleo y por el sector financiero en la curva de rendimiento de los treasuries y la baja tasa de interés de referencia de los fondos federales, son aspectos que aún persisten en la economía.

\section{REFERENCIAS BIBLIOGRÁFICAS}

Bloomberg / Nueva York, entre 1 y 3 p.m. Consulta: 12-10-11

Hilsenrath, J y Brian Blackstone; 2009; La Fed se aferra a su agresiva estrategia». The Wall Street Journal, 30 de abril de 2009.

Lotta, R.; 2008; El derrumbe financiero y la locura del imperialismo, Revolución \#127, 20 de abril de 2008, http://rwor.org/a/127/EconomyMeltdown-es.html\#text3.

Navarro, E; Cómo Entender la Crisis Financiera actual de los Estados Unidos y por qué se ha convertido en un problema global, Revista la Moneda, Banco Central de Reserva del Perú http://www.mtc.gob.pe/portal/consultas/cid/Boletines_CID/41_ ENERO/pdf/otras/crisis.pdf. 


\section{Carlos Palomino Selem}

Gráfica N. 1: Hoja de Balance del Fed

\section{Federal Reserve Balance Sheet}

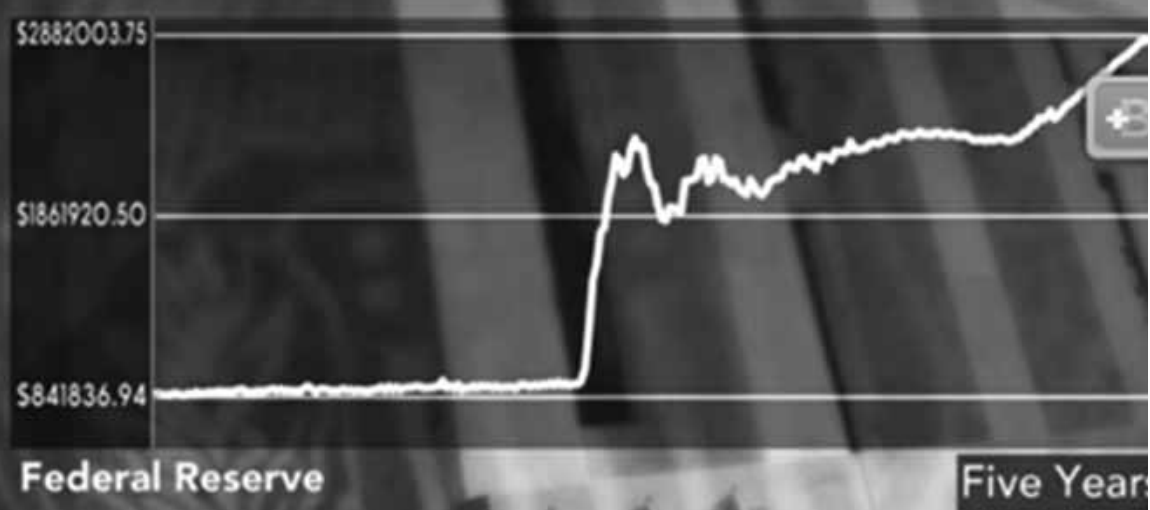

Fuente: Bloomberg / Nueva York, entre 1 y 3 p.m.. Consulta: 12-10-11 